\title{
NVO study of Super Star Clusters in nearby galaxies
}

\author{
Bradley C. Whitmore \\ Space Telescope Science Institute, 3700 San Martin Dr., Baltimore, MD, 21218, USA \\ email:whitmore@stsci.edu
}

We are using NVO tools to obtain a large $(\mathrm{N} \simeq 100)$, uniform (SDSS images, cross checked with HST images; analyzed using WESIX) database of super star clusters in nearby star-forming galaxies in order to address two fundamental astronomical questions: (i) Is the initial luminosity function of star clusters universal?; and (ii) What fraction of super star clusters is 'missing' in optical studies (i.e., are hidden by dust)?

It is now well established that large numbers of young massive star clusters (i.e., 'Super Star Clusters'; hereafter SSCs) form in gas-rich mergers and starburst galaxies (e.g., see review by Whitmore 2003, in A Decade of HST Science, eds. M. Livio, et al., Cambridge: CUP, 153). Larsen \& Richtler (2000, A\&A, 354, 836) have shown that compact young clusters form in normal spiral galaxies as well, with the same luminosity function found for the clusters in merging galaxies. The difference is that these clusters are generally much fainter and less massive than their counterparts in merging systems. These studies have sparked an on-going discussion concerning the question of whether star formation in violent environments (i.e., in mergers and starbursts) is fundamentally different from star formation in quiescent settings (i.e., in spirals).

Whitmore (2003) addressed this question by making a simple plot of the brightest cluster versus the number of clusters in a galaxy, which suggested a universal rather than bimodal physical process. Our program is designed to readdress this issue using a more uniform database and analysis procedure.

Another important aspect of this project is to quantify the fraction of SSCs which are 'missing' (i.e, seen in the near infrared by 2MASS but not in optical studies; see Whitmore \& Zhang; 2002, AJ, 124, 1418). This will also allow us to address the question of whether the fraction of obscured SSCs differs for mergers and starbursts when compared with normal spirals. We are also interested in determining whether HST preview images can be used to do serious science (i.e., what is the agreement between magnitudes determined from SDSS, HST observations, and HST preview images)? At present, this looks quite promising, with uncertainties of $\simeq 10 \%$. While this is large by normal photometric standards, for the purposes of this study this might be quite sufficient.

We plan to use two independent approaches for sample selection: (1) a volume limited sample, and (2) galaxies selected to provide a roughly uniform distribution in $\log (\mathrm{N})$. The primary NVO tools that we use for the project are: WESIX, Aladin, VOTool, and OpenSKYQuery.

\section{Acknowledgements}

I would like to acknowledge my co-investigators on this project: Kevin Lindsay, Rupali Chandar, Chris Hanley, and Ben Chan. This project is supported by a NVO Research Initiative grant. 